\title{
FORMATION AND ANNEALING OF MICROPORES IN HEMATITE
}

\author{
C. Dauwe, G.M. Da Costa*, T. Van Hoecke and D. Segers \\ Section Subatomic and Radiation Physics, University of Gent \\ Proeftuinstraat 86, 9000 Gent, Belgium
}

In iron oxide powders positrons are trapped in the surface layers and the value of the $S$-parameter is an indicator of the total specific surface, including external surface and internal surfaces of micropores. The evolution of micropores is followed when $\alpha-\mathrm{FeOOH}$ is thermally decomposed into $\alpha-\mathrm{Fe}_{2} \mathrm{O}_{3}$. It is shown that the micropore formation is not linked to the decomposition process, but that they also form in hematite synthesized from a ferrihydrite precursor in aqueous solution.

PACS numbers: $78.70 . B j$

\section{Introduction}

Hematite particles can be synthesized by various methods, yielding particles with different shapes, crystallinity and defect structures. One of the most popular methods is the one described by Schwertmann and Cornell [1] where particles of goethite, $\alpha-\mathrm{FeOOH}$, are precipitated from an aqueous solution of $\mathrm{Fe}\left(\mathrm{NO}_{3}\right)_{3}$. The goethite particles are then thermally decomposed into hematite $\alpha-\mathrm{Fe}_{2} \mathrm{O}_{3}$ by a topotactic reaction in air or in vacuum. Measurements of the specific surfaces by means of nitrogen adsorption at $77 \mathrm{~K}$ (BET and de Boer $t$-plot techniques), as well as electron microscopy have revealed that the thermal transformation in vacuum [2] and in air [3] between $200^{\circ} \mathrm{C}$ and $300^{\circ} \mathrm{C}$ of acicular goethite yields hematite with slit-shaped micropores, which are replaced by spherical mesopores in the temperature region $300^{\circ} \mathrm{C}$ to $600^{\circ} \mathrm{C}$. Above $600^{\circ} \mathrm{C}$ interparticle sintering takes place and nonporous or "defect-free" hematite is obtained.

A first examination of hematite powders with positron annihilation showed that in contrast to alumina, magnesia and silica powders, no interparticle positronium is formed [4] and that as a consequence the usual positronium-surface interaction [5] cannot be used as a surface probe. On the other hand, positrons are expected to be trapped at point defects and extended defects, and particularly at surfaces and the usual $S$-parameter should give indication of the creation and annihilation of the different types of pores. Brazil.

*On lea ve from Departamento de Quimica,Universidade Federal de Ouro Preto, Minas Gerais, 


\section{Experimental details}

The preparation of the goethite sample is fully described in Ref. [1], p. 64. The X-ray diffraction (XRD) of the sample obtained showed it to be pure goethite with appreciable line broadening due to the small size of the particles. The mean dimensions inferred from this line broadening are $\mathrm{MCD}_{111} \approx 330 \AA, \mathrm{MCD}_{110} \approx$ $240 \AA$. In the case of goethite none of these directions coincide with any external dimension of the particles, but. electron micrography confirm an acicular shape with approximately $300 \AA$ smallest dimension and $3000-4000 \AA$ longest dimension. Thermogravimetry in nitrogen gas flow and at $5 \mathrm{~K} / \mathrm{min}$ heating rate on this sample shows a $12.3 \mathrm{wt} \%$ loss corresponding to the $2 \mathrm{FeOOH} \rightarrow \mathrm{Fe}_{2} \mathrm{O}_{3}+\mathrm{H}_{2} \mathrm{O}$ transformation starting at $200^{\circ} \mathrm{C}$ and ending at $300^{\circ} \mathrm{C}$.

Two cylindrical samples of $15 \mathrm{~mm}$ diameter and 2-3 mm thick were pressed with a pressure of $2.8 \times 10^{7} \mathrm{~Pa}$ to constitute a sandwich with a Na-22 source on kapton foil. The sandwich was inserted into a vacuum chamber, and the temperature could be regulated between $100 \mathrm{~K}$ and $600 \mathrm{~K}$. The normal operating vacuum is $5 \times 10^{-5} \mathrm{~Pa}$.

The $S$-parameter spectrometer is a standard HPGe detector with preamplifier, main amplifier, biased amplifier and a Canberra model 40 multichannel analyzer with two peak stabilizers. For the stabilization an own made highly stable double pulser injects zero- and gain-pulses into the test input of the preamplifier. The $S$-parameter is taken as integration over the central 41 channels of the annihilation line. Compton, peak, and high integration regions are set in order to calculate a Compton-to-peak ratio $C / R$ and to monitor a possible change in three-quantum annihilation contribution.

\section{Results and discussion}

\subsection{The goethite-hematite decomposition}

The goethite sample is introduced into the chamber and an $S$-parameter isotherm is taken at room temperature (RT) in air. The mean value in these conditions is $S_{\text {air }}=(0.5234 \pm 0.0007)$. Then the vacuum system is started and it is observed that at RT the desorption of water from the surface causes an increase in $S$ which stabilizes after 2 hours (see Fig. 1$)$ at the value $S_{\text {vac }}=(0.5296 \pm 0.0007)$. The value $S_{\text {air }}$ is used as a normalizing value in the presentation of these data. The fact that the $S$-parameter is sensitive to the desorption of water indicates that the positrons probe the surface layers, but at the same time there is not the slightest change in $C / R$ which indicates that no three-quantum annihilation is involved, and thus no Ps is ejected from the particles. Therefore we attribute the increase in $S$ to positrons trapped at the surface, both outer and inner and we consider the $S$-parameter to be linear function of the specific surface.

Next, the temperature is increased from $300 \mathrm{~K}$ to $600 \mathrm{~K}$ in steps of $5 \mathrm{~K}$ and a $15 \mathrm{~min}$ measurement is taken at each temperature. When reaching $600 \mathrm{~K}$ this temperature is hold for an isotherm of 20 consecutive measurements, and then the temperature is lowered again to $300 \mathrm{~K}$ as in the first part. Figure 2 shows the $S$-parameter in warming and cooling cycles, where this parameter is normalized to the mean value $S_{\text {vac }}$, obtained before. During all cycles and treatments no 

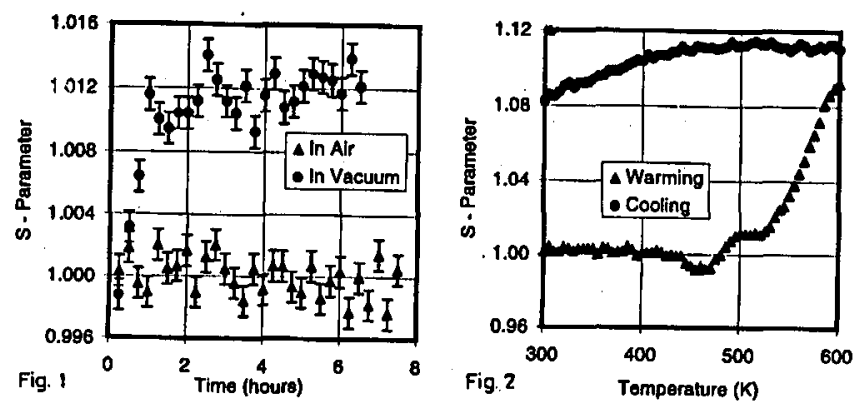

Fig. 1. Goethite; $S$-parameter in air at $300 \mathrm{~K}$ and the effect of desorption of water in vacuum.

Fig. 2. Decomposition of goethite particles into hematite, with creation of micropores between 500 and $600 \mathrm{~K}$.

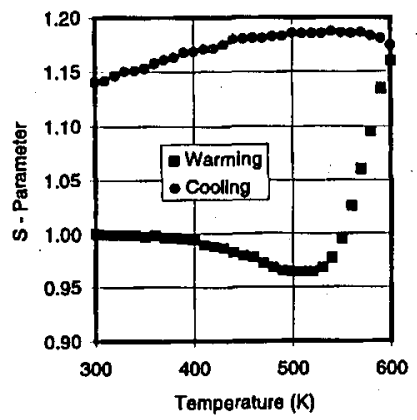

Fig. 3. Growth at $600 \mathrm{~K}$ of stable micropores in hematite from goethite precursor.

changes were observed in $C / P$ and we will not give any further thoughts to this absence of Ps. In the warming cycle in Fig. 2 we observe no appreciable change in $S$ between $300 \mathrm{~K}$ and $400 \mathrm{~K}$, which confirms that all water was desorbed at RT in vacuum. At $440 \mathrm{~K}$ a slight decrease in $S$ probably indicates the onset of the goethite-to-hematite decomposition but at $460 \mathrm{~K}$ it starts to rise again to reach eventually a small plateau between 500 and $520 \mathrm{~K}$. These minute details between $440 \mathrm{~K}$ and $520 \mathrm{~K}$ have never been observed by BET methods for the specific surface and it is not yet clear if these are reproducible features. At $520 \mathrm{~K}$ there is the onset of a huge increase in the $S$-parameter which illustrates the increase in specific surface due to the micropores generated by the thermal decomposition. According to the thermogravimetric results this decomposition is completed at $570 \mathrm{~K}$. Nevertheless, we observe that the $S$-parameter still increases between 570 and $600 \mathrm{~K}$. The isotherm at $600 \mathrm{~K}$ shown in Fig. 3 indicates that the micropores still grow to eventually stabilize after two hours. Upon cooling again to $300 \mathrm{~K}$ the $S$-parameters show a smooth and reversible variation and we must assign this curve to hematite with stable micropores. 


\subsection{Hematite and micropores}

It may be argued that the observed increase in $S$ is only due to the difference in crystal structure between goethite and hematite, without the need of micropores. Therefore we prepared a hematite sample from goethite by heating at $600 \mathrm{~K}$ in air for 4 hours. According to Rendon et al. [3] this should produce a hematite where the micropores have disappeared by internal sintering, yielding spherical mesopores with a much lower specific surface. Nevertheless, as we just observed the stability of micropores at $600 \mathrm{~K}$ in vacuum, this annealing temperature seems to be too low. However, when comparing micropore formation in air and in vacuum it seems that in vacuum they are slightly more stable, and by a strike of good luck $600 \mathrm{~K}$ in air was sufficiently high to anneal the micropores. Whether mesopores are present or not might not be relevant here if the mesopores surfaces are not efficiently observed by the positrons. Therefore the new sample may be considered as defect-free hematite. On this sample the same heating-isotherm-cooling cycle was applied and the evolution of the $S$-parameter is shown in Fig. 4, where all $S$ values are normalized on the value at $300 \mathrm{~K}$ after evacuation. The systematics shown in Fig. 4 are basically the same as in Fig. 2 and the magnitude of the micropore peak is even higher, although no decomposition or phase transition takes place. This proves that

1) the initial state is free of micropores and

2) the micropores are generated again between $500 \mathrm{~K}$ and $600 \mathrm{~K}$ without the need of a goethite decomposition. This is strong indication that the micropore formation is not linked to the goethite precursor of the hematite, but is an intrinsic property of hematite between $500 \mathrm{~K}$ and $600 \mathrm{~K}$.

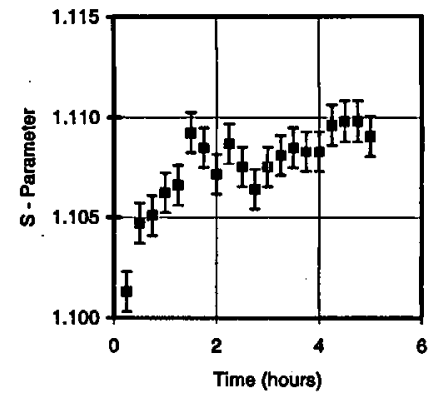

Fig. 4. Formation of new micropores in annealed hematite with goethite precursor.

\subsection{Stability of micropores}

In order to prove that micropore creation is not linked to the goethite precursor, a third hematite sample was prepared from a ferrihydrite precursor according to the method described in Ref. [1], p. 103, method 4. By this method we obtain diamond shaped particles with XRD dimensions $\mathrm{MCD}_{012}=600 \AA$, $\mathrm{MCD}_{104}=400 \AA, \mathrm{MCD}_{110}=600 \AA$. The warming-up curve for this sample in 

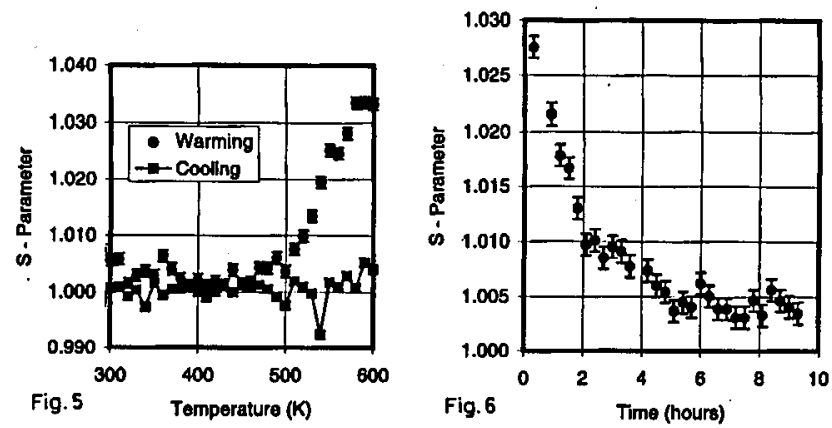

Fig. 5. Formation of micropores in hematite prepared from a ferrihydrite precursor. Fig. 6. Isothermal annealing at $600 \mathrm{~K}$ in vacuum of micropores in hematite with ferrihydrite precursor.

Fig. 5 clearly shows the formation of micropores starting at $500 \mathrm{~K}$ and peaking at $580 \mathrm{~K}$, although the magnitude of the increase in specific surface is much lower than in the previous cases. Surprisingly, the cooling curve after the usual isotherm at $600 \mathrm{~K}$ shows no structure at all and is situated at the value of pore-free initial hematite. The reason for this is illustrated in the isotherm of Fig. 6 where the micropores are seen to be unstable at $600 \mathrm{~K}$ and to disappear after approximately 4 hours. As a result we can conclude that the stability of the micropores probably depend on a number of parameters such as precursor, particle size and shape, atmosphere of annealing, but that the region between $580-620 \mathrm{~K}$ is where most micropores anneal by internal sintering.

\section{Acknowledgments}

One of us (G.M.D.C.) acknowledges financial support from the CNPq.

\section{References}

[1] U. Schwertman, R.M. Cornell, Iron Oxides in the Laboratory, VCH, Weinheim 1991.

[2] H. Naono, R. Fujiwara, J. Colloid Interface Sci. 73, 406 (1980).

[3] J.L. Rendon, J. Cornejo, P. De Arambarri, C.J. Serna, J. Colloid Interface Sci. 92, 508 (1983).

[4] C. Dauwe, unpublished results.

[5] C. Dauwe, Mbungu Tsumbu, Phys. Rev. 45, 9 (1992). 